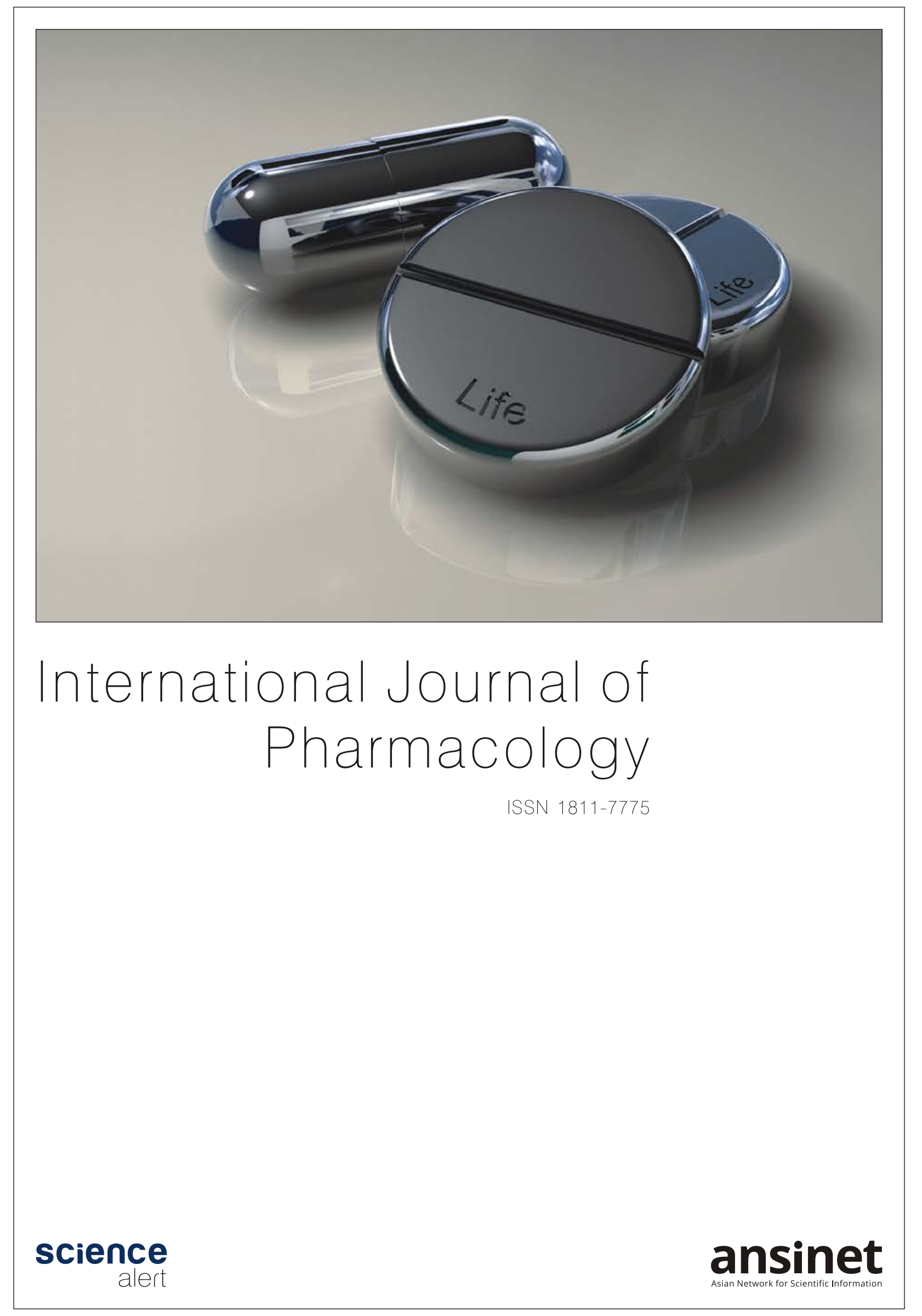




\title{
Research Article Protective Effect of Naringenin Against Isoniazid-induced Adverse Reactions in Rats
}

\author{
${ }^{1}$ Osama Abdel-Ghaffar, ${ }^{1}$ Atef Abdel-Moneem Ali and ${ }^{2}$ Sara Abdel-Mohsen Soliman \\ ${ }^{1}$ Division of Physiology, Department of Zoology, Faculty of Science, Cairo University, Giza, Egypt \\ ${ }^{2}$ National Research Center (NRC), Dokki, Giza, Egypt
}

\section{Abstract}

Background and Objective: Isoniazid (INH) has a vital role in the treatment of tuberculosis. Nevertheless, the INH-induced hepatotoxicity was documented. Naringenin (NGN) is a dietary flavonoid that has antioxidative and anti-inflammatory properties. The current study aimed to evaluate the protective role of NGN against INH-induced toxicity in male Wister rats. Materials and Methods: One hundred rats were randomly assigned to four equal groups, the control, INH-treated, (INH+NGN)-treated and NGN-treated. Certain hematological parameters, hepatic function markers, lipid and protein profiles in serum as well as the lipid peroxidation and endogenous antioxidants content in the liver were analyzed. The INH and NGN were orally administered at dose levels of 54 and $50 \mathrm{mg} \mathrm{kg}^{-1} \mathrm{~b} . w \mathrm{w}$., respectively. Statistical analysis was carried out using two-way ANOVA, least significant differences (LSD), Duncan's test and correlation analysis. Results: The INH-treated rats showed a significant decline in the studied hematological parameters, the serum levels of high-density lipoprotein (HDL), albumin (A) as well as the hepatic levels of glutathione (GSH) and activities of catalase (CAT), superoxide dismutase (SOD) and glutathione reductase (GR). This was accompanied by a significant elevation in the levels of total lipids (TL), triglycerides (TG), total cholesterol (TC), low-density lipoproteins (LDL), globulin (G), total bilirubin (TBil) and the activities of alanine and aspartate aminotransferase (ALAT and ASAT) and alkaline phosphatase (ALP) as well as the hepatic levels of malondialdehyde (MDA). In contrast, the administration of NGN $1 \mathrm{~h}$ prior to INH notably improved all the studied parameters. The rats administered NGN alone showed insignificant differences in all the studied parameters with the controls except for marked reductions in the levels of TL, TC and LDL. Conclusion: This study disclosed that INH administration to Wistar rats, at a high therapeutic dose level, could induce a hepatic injury in addition to certain hematologic and metabolic alterations. Yet, the pretreatment with NGN could ameliorate these alterations via its antioxidative effect.

Key words: Anti-TB drugs, isoniazid, INH-induced hepatotoxicity, naringenin, oxidative stress, hepatotoxicity, anemia, antioxidants, flavonoid benefits 


\section{INTRODUCTION}

Tuberculosis (TB) is one of the most serious infectious diseases especially in the developing countries ${ }^{1}$. The TB is caused by a certain type of bacteria known as Mycobacterium tuberculosis (M. tuberculosis) ${ }^{2}$. In 1952, isoniazid (isonicotinic acid hydrazide, $\mathbf{I N H}$ ) was used for the first time as an antitubercular (anti-TB) chemotherapeutic agent with high efficacy due to its low minimum inhibitory concentration against $M$. tuberculosis ${ }^{3}$. Additionally, INH has an effective role not only in the treatment but also in the prevention of TB'. Unfortunately, the treatment regimens using $\mathrm{INH}$ were accompanied by adverse side effects such as hepatotoxicity ${ }^{5}$. In the liver, INH is biotransformed into hydrazine $(\mathrm{HZ})$ which is the reactive metabolite ${ }^{6}$. The $\mathrm{HZ}$-induced hepatotoxicity was attributed to its ability to cause oxidative stress through overproduction of free radicals including hydrogen peroxide $\left(\mathrm{H}_{2} \mathrm{O}_{2}\right)$ as well as protein carbonyl ${ }^{7}$. The resultant $\mathrm{H}_{2} \mathrm{O}_{2}$ can interfere with the mitochondrial functions through inhibiting succinate dehydrogenase ${ }^{8}$ leading to reduced production of adenine triphosphate (ATP) ${ }^{9}$.

Recent studies were targeted to investigate the potential hepatoprotective role of some natural extracts depending on their antioxidant properties. Eminzade et al. ${ }^{10}$ found that the extract of Silybum marianum seeds has a hepatoprotective action on rat liver against hepatotoxicity induced by antitubercular agents. However, the authors recommended further high-quality placebo-adjusted trials prior to its application in human. In addition, Basheer et al. ${ }^{11}$ reported the ameliorative role of fish oil extract on the hepatotoxic effects of INH and rifampicin (RMP) in rats.

Flavonoids are active phytochemicals that have promising protective roles against many health threatening issues ${ }^{12}$. Naringenin (NGN) is a dietary flavonoid that is abundant in orange, grapefruit and tomato $^{13}$. NGN also showed anticarcinogenic, anti-inflammatory ${ }^{14}$, antioxidant ${ }^{15}$, hypolipidemic ${ }^{16}$ and hypoglycemic effects ${ }^{17}$ on the biological systems. The flavonoid NGN exhibited hepatoprotective effects against liver injury caused by $\mathrm{CCl}_{4}$ in mice ${ }^{18}$ as well as arsenic-induced hepatotoxicity in rats ${ }^{19}$. However, there is no in vivo study was executed till now to show the potential protective role of $\mathrm{NGN}$ against $\mathrm{INH}$-induced hepatotoxicity.

Thus, the present study was subjected to study the possible protective role of NGN against the INH-induced adverse effects on certain hematological criteria, serum biochemical and hepatic oxidative stress parameters, in male albino rats.

\section{MATERIALS AND METHODS}

This study was carried out 2016, in the labs of Department of Zoology, Faculty of Science, Cairo University, Giza and in the National Research Center, Dokki, Giza, Egypt.

Chemicals: The INH (CAS No. 54-85-3, purity $\geq 99 \%$ ) and NGN (CAS No. 67604-48-2, purity $\geq 95 \%$ ) were purchased from Sigma-Aldrich Co, St. Louis, MO, USA. All the kits used for oxidative stress biomarkers, antioxidants and biochemical parameters were obtained from Bio-diagnostics (Dokki, Giza, Egypt).

Experimental model: Adult healthy male Wistar rats, Rattus norvegicus, weighting 120-150 g were obtained from the Animal House of National Organization for Drug Control and Research (NODCAR), Agouza, Giza, Egypt. Animals were housed in clean polyacrylic cages in the Animal House of Zoology Department, Faculty of Science, Cairo University, with five animals/cage, supplied with standard feed and water ad libitum. The rats were kept in conventional conditions of $12 / 12 \mathrm{~h}$ light-dark cycle and at room temperature $\left(22-25^{\circ} \mathrm{C}\right)$. Prior to the onset of the main experiments, rats were allowed to acclimatize for 2 weeks in the animal house.

Ethical approval: The applied procedures, in the present study, were approved by Cairo University Institutional Animal Care and Use Committee (CU-IACUC), Egypt under the IACUC Permit Number of CU/I/S/6/16 at April 2016. The animals were handled in accordance with international guidelines for the care and use of laboratory animals.

Experimental design: Randomly, one hundrad rats were divided into four equal groups (control, INH-treated, (NGN+INH)-treated and NGN-treated) with twenty-five individuals/group. Rats of the control group received $1 \%$ carboxymethyl cellulose (CMC). The INH-administered group was orally administered a daily dose of $54 \mathrm{mg} \mathrm{kg}^{-1}$ b.wt., of INH (in 1\% CMC) for 5 weeks. The NGN-administered group orally received a daily dose of $50 \mathrm{mg} \mathrm{kg}^{-1}$ b.wt., of NGN (in $1 \%$ of CMC) for 5 weeks. The doses of INH and NGN were assigned according to Abdel-Ghaffar et al. ${ }^{20}$ and Renugadevi and Prabu ${ }^{21}$, respectively. The rats of the fourth group were orally administered a $50 \mathrm{mg} \mathrm{kg}^{-1}$ b.wt., of NGN $1 \mathrm{~h}$ prior to the administration of $\mathrm{INH}\left(54 \mathrm{mg} \mathrm{kg}^{-1}\right.$ b.wt.) for 5 weeks. 
Sampling: At the end of each experimental week, five rats from each group were collected. Rats were euthanized using $50 \mathrm{mg} \mathrm{kg}^{-1}$ of sodium pentobarbital and were sacrificed. Blood samples were divided into two parts. The first part was collected on EDTA for haematological analyses. The second part was collected into dry test tubes and then centrifugated at $3000 \mathrm{rpm}$ in order to separate serum. The sera were kept at $-20^{\circ} \mathrm{C}$ for further biochemical analysis. In order to collect the hepatic tissue, rats were immediately dissected. The liver was homogenized with $10 \% \mathrm{w} / \mathrm{v}$ ratio in ice-cold $50 \mathrm{mM}$ Tris $\mathrm{HCl}$ buffer at pH 7.4 and then centrifuged at 10,000 rpm for $20 \mathrm{~min}$ at $4{ }^{\circ} \mathrm{C}$. The supernatant was collected and kept in deepfreeze at $-20^{\circ} \mathrm{C}$ for further analyses.

Estimation of hematological parameters: The hematological parameters including red blood cell (RBC) count, white blood cell (WBC) count, platelet (PLT) count, hemoglobin ( $\mathrm{Hb}$ ) content and packed cell volume (PCV) were analyzed using Medonic M-Series analyzer (Clinical Diagnostics solutions Inc, Florida, USA).

Estimation of serum biochemical parameters: In the serum of all the experimental groups, the levels of total lipids ( $T L$ ), total cholesterol (TC), triglycerides (TG), low density lipoprotein cholesterol (LDL-C), high density lipoprotein cholesterol (HDL-C), total proteins (TP), albumin (A), globulin (G), aspartate aminotransferase (ASAT), alanine aminotransferase (ALAT), alkaline phosphatase (ALP), total bilirubin (TBil) and direct bilirubin (DBil) were measured colorimetrically using Biodiagnostics kits (Dokki, Giza, Egypt).

Lipid peroxide assay: The level of malondialdehyde (MDA) in the liver homogenate was assayed according to the technique described by Ohkawa et al.22. The principle of this method depends on the reaction of the liberated MDA after lipid peroxidation (LPO) of the cell membranes with thiobarbituric acid in acidic medium.

Non-enzymatic and enzymatic antioxidant assay: The concentrations of non-enzymatic (glutathione, GSH) as well as enzymatic (catalase, CAT, superoxide dismutase, SOD, glutathione reductase, $G R$ ) antioxidants were estimated in the homogenate of the liver of control male albino rats and those administered with INH and/or NGN. The method by which GSH content was measured was based on the reaction of 5,5'Dithiobis-2-nitrobenzoic acid with $\mathrm{GSH}^{23}$. The CAT activity was estimated in accordance to the method described by Aebi $^{24}$. The SOD activity assessment was based on the ability of SOD to inhibit the reduction reaction of nitroblue tetrazolium dye mediated by phenazine methosulphate ${ }^{25}$. The principle for measuring the GR activity was based on its ability to catalyze the reduction of glutathione (GSSG) as described by Goldberg and Spooner ${ }^{26}$.

Statistical analysis: Data were statistically analyzed by the aid of Statistical Package of the Social Sciences, SPSS version 23 (copyrighted by IBM SPSS software, USA). Two-way analysis of variance (ANOVA) was used to study the effect of the type of treatment (control, INH alone, NGN alone and INH+NGN), the experimental periods (1, 2, 3, 4 and 5 weeks) and their interaction together on all the studied parameters. Duncan and least significant difference (LSD) tests were utilized to examine the similarities and differences among all the experimental groups and periods. Correlation coefficient analysis was applied to study the relationships between the studied variables and the experimental durations. Data were expressed as a mean \pm standard error of mean (SEM).

\section{RESULTS}

Effect on hematological parameters: The results of hematological parameters (Table 1) revealed that the type of treatment significantly affected all the studied blood parameters except for the PLT count that did not show any significant differences among all the studied groups. However, the experimental time exhibited marked effects only on the $\mathrm{Hb}$ content. Rats of INH-administered group showed a notable decline in the RBC and WBC counts, Hb content and PCV, as compared to the controls. The experimental time was inversely correlated with the reduction in the $\mathrm{WBC}, \mathrm{RBC}, \mathrm{Hb}$ and PCV of INH-treated group. As compared to the rats of INH-treated group, the rats administered NGN $1 \mathrm{~h}$ prior to the INH exhibited significant elevations in the RBC, WBC counts, $\mathrm{Hb}$ content and PCV, throughout the treatment periods. In comparison to the corresponding controls, all the studied hematological parameters of rats administered with NGN followed by INH did not show any significant differences throughout the experiments. In the (NGN+INH)-administered group, the counts RBC, WBC, Hb content and PCV exhibited moderate positive correlations with the experimental time. The rats administered with NGN alone showed similarities in all the blood parameters with those of the controls, at all the experimental intervals. There were moderate positive correlations between the experimental time and the counts of RBC, WBC, Hb levels and PCV of the rats administered NGN alone. 


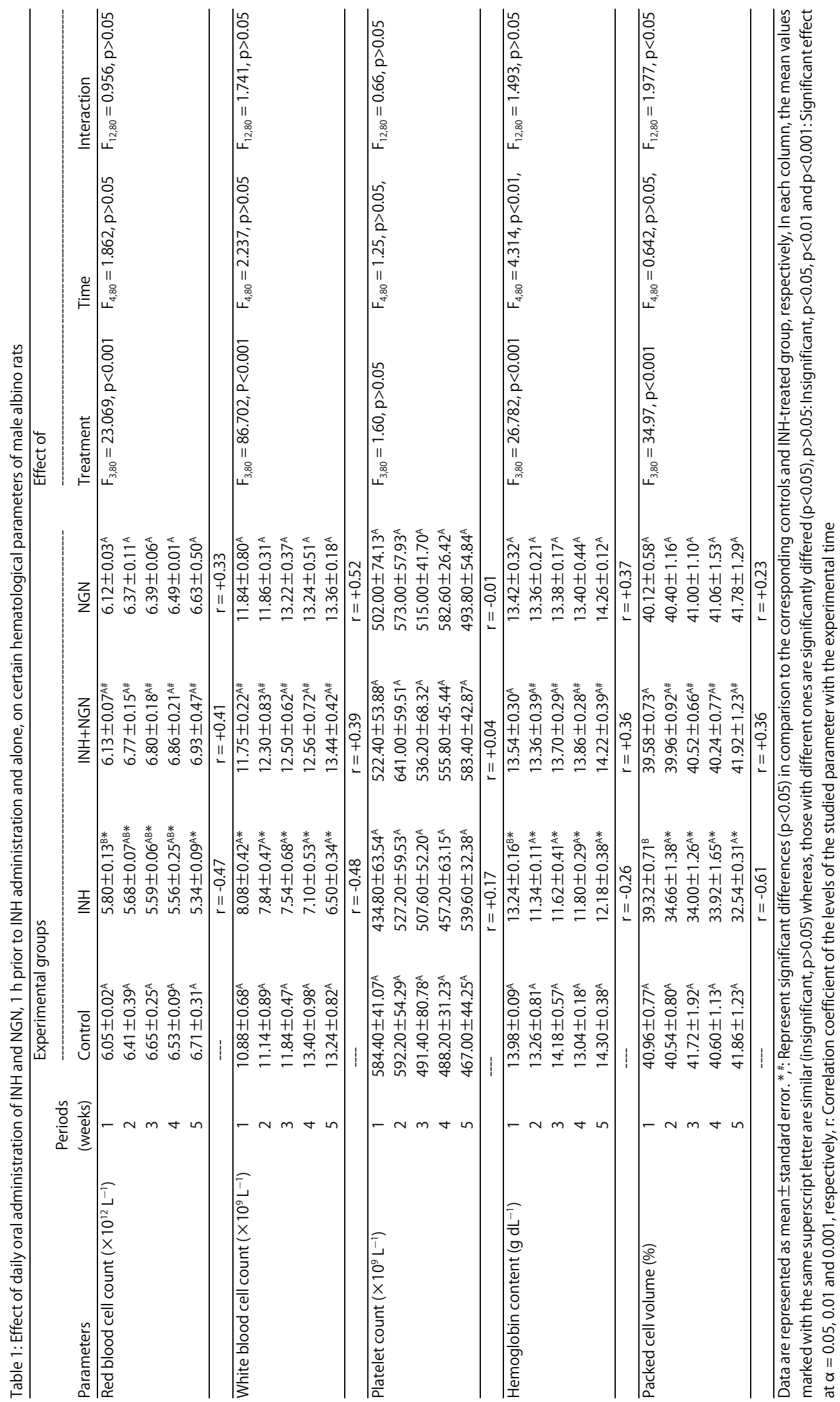


Effect on serum biochemical parameters: The lipid profile of the experimental animals as affected by the administration of INH, INH+NGN and NGN are shown in the Table 2. The serum levels of TL, TC, TG, LDL-C and HDL-C of the rats were markedly influenced by the type of treatment. However, the experimental periods remarkably affected the levels of TC, TG and LDL-C only. In comparison to the control, all the studied lipid profile parameters of $\mathrm{INH}$-treated group were significantly elevated except the levels of HDL-C that were notably reduced, throughout the experiments. In the INH-treated rats, the experimental time was correlated positively with the levels of $\mathrm{TL}, \mathrm{TC}, \mathrm{TG}$ and $\mathrm{LDL}-\mathrm{C}$ but negatively with the levels of $\mathrm{HDL}-\mathrm{C}$. The rats treated with NGN before INH exhibited a marked reduction in the levels of $T L$, TC, TG and LDL-C, as compared with the INH-treated rats, at all the experimental time intervals except the insignificant changes in the levels of TG at the 1st and 2nd weeks. However, the levels of HDL-C of rats administered with NGN followed by INH showed significant increase as related to the INH-treated group, at all the experimental intervals. Moreover, the treatment with NGN followed by INH retrieved the levels of lipid variables to the normal values of the controls. In the (NGN+INH)-treated rats, the experimental time showed weak correlations with the levels of all the studied lipid parameters except the strong negative correlation with the levels of $L D L-C$ as well as the moderate positive correlation with the HDL-C levels. From the 2 nd week of treatment with NGN alone, the serum levels of TL, TC and LDL-C were significantly declined till the end of the experiments, as compared to the controls. The reductions in the levels of TL, TC and LDL-C of NGN-treated rats were negatively correlated with the experimental periods. However, the concentrations of TG and HDL of rats-administered NGN alone were not significantly $(p>0.05)$ differ from those of the corresponding controls throughout the experiments.

Serum protein profile of rats (Table 3 ) was noticeably affected by the type of treatment but not with the time except for the serum levels of TP that were homogenous among all the experimental groups. Rats administered INH alone exhibited marked reductions in the levels of albumin simultaneous with a significant increase in the levels of globulin, as compared to the controls, at all the time intervals. Thus, the A/G ratio of INH-treated rats was remarkably reduced. The time factor exhibited negative correlations with albumin levels and $A / G$ ratio but positive correlations with globulin levels in INH-administered rats. On the other hand, the rats of $(\mathrm{NGN}+\mathrm{INH})$-treated group displayed a marked increase in the levels of albumin and $A / G$ ratio but a marked decrease in the levels of globulin, as compared to the $\mathrm{INH}$-treated group. All the protein profile parameters of the rats administered NGN prior to $\mathrm{INH}$ were not significantly different ( $p>0.05$ ) from those of the controls at all time intervals except a marked decrease in albumin levels at the 1 st and 2 nd weeks. The rats administered NGN alone did not exhibit any significant changes in the levels of the protein profile parameters, as compared to the controls during the whole period of experiments. In both the NGN-treated and $\mathrm{NGN+INH-treated} \mathrm{rats,} \mathrm{the} \mathrm{experimental} \mathrm{time} \mathrm{correlated}$ positively with the levels of albumin while negatively with the levels of globulin.

Liver function markers, as influenced by the administration of $\mathrm{INH}$ and NGN, alone and mixed, were presented in Table 4. The activities of ASAT, ALAT and ALP, in serum of rats were significantly affected by the type of treatment and time factor. The serum levels of TBil were significantly affected by the type of treatment only whereas the serum levels of DBil were not affected by any of the studied factors. In comparison to the controls, the INH-treated rats showed significant elevations in the activities of ASAT and ALAT, at all the experimental intervals. The activity of ALP in the INH-treated rats was similar to those of the controls at the 1 st and 2nd weeks but were markedly increased at the rest of the experimental intervals. From the 2 nd week till the end of the experiments, the levels of TBil of INH-treated rats were significantly increased as referred to the controls. The levels of DBil of INH-administered rats did not differ significantly ( $p>0.05$ ) from those of the controls. In the INH-treated rats, the activities of ASAT and ALAT were directly correlated with the experimental intervals. The activities of ASAT and ALAT of (NGN+INH)-administered rats were markedly lower than those of the INH-treated group at all the experimental periods except the insignificant decrease in the ALAT activity at the 1st week. In the rats of (NGN+INH)-treated group, the ALP activity and TBil level started to decline significantly from the $3 r d$ week till the end of the experiments, as compared to the INH-treated group. The activities of ALP, ASAT and ALAT as well as the levels of TBil and DBil of (NGN+INH)-treated rats were not significantly different $(p>0.05)$ from those of the controls, at all time intervals, except a significant increase in the activity of ALAT at the 1st week. The experimental time showed negative correlations with the activities of ASAT, ALAT and ALP in the rats administered NGN prior to INH. The administration of NGN alone did not cause any significant change in the activities of aminotransferases and ALP or in the levels of TBil and DBil, as compared to the controls, during the whole experimentation period.

\section{Effect on the hepatic lipid peroxidation and endogenous antioxidants: The effects of INH, NGN+INH and NGN} administration on the levels of hepatic MDA and GSH and the activities of endogenous antioxidant enzymes were shown in Table 5. The hepatic levels of MDA and GSH as well as the 


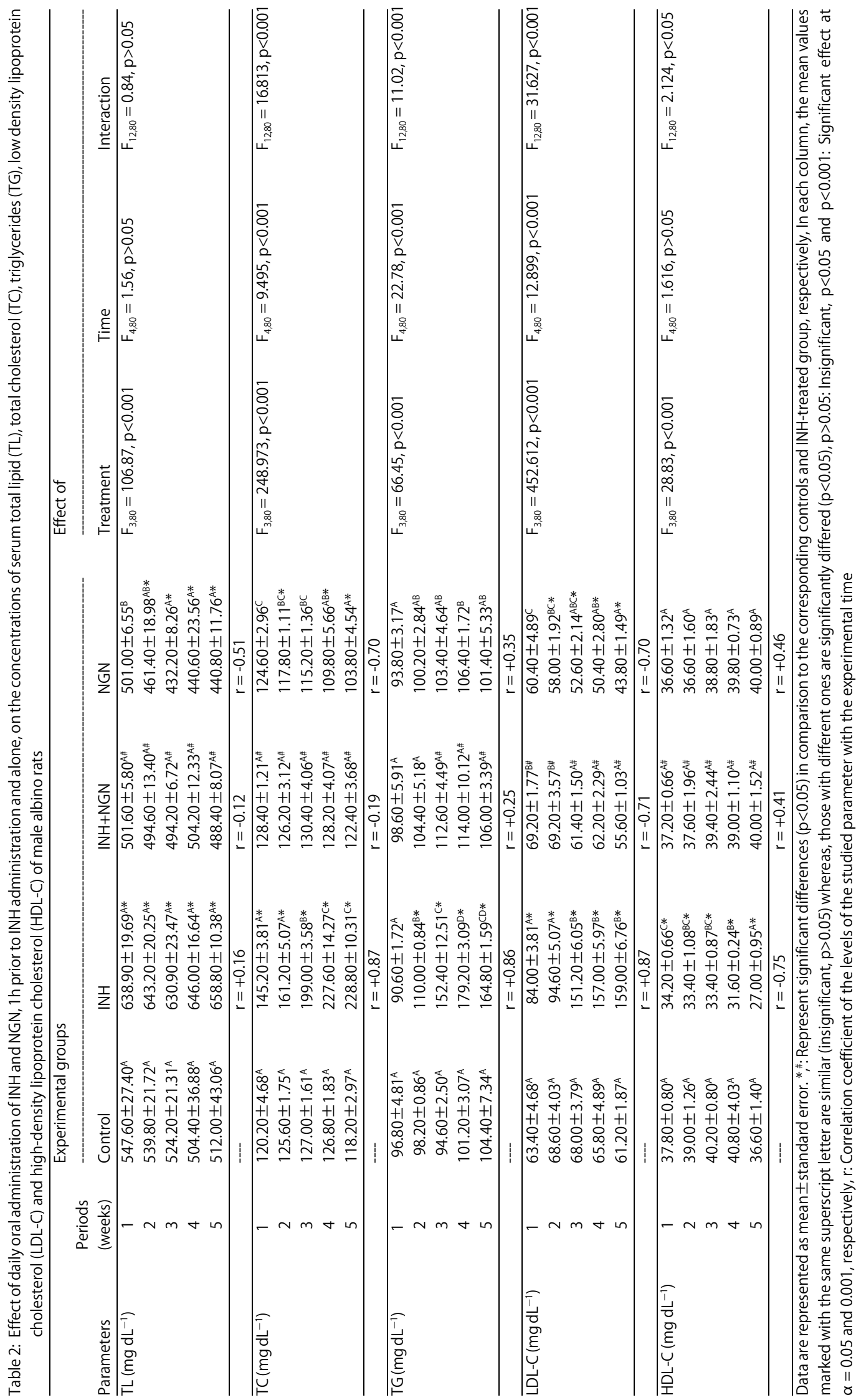




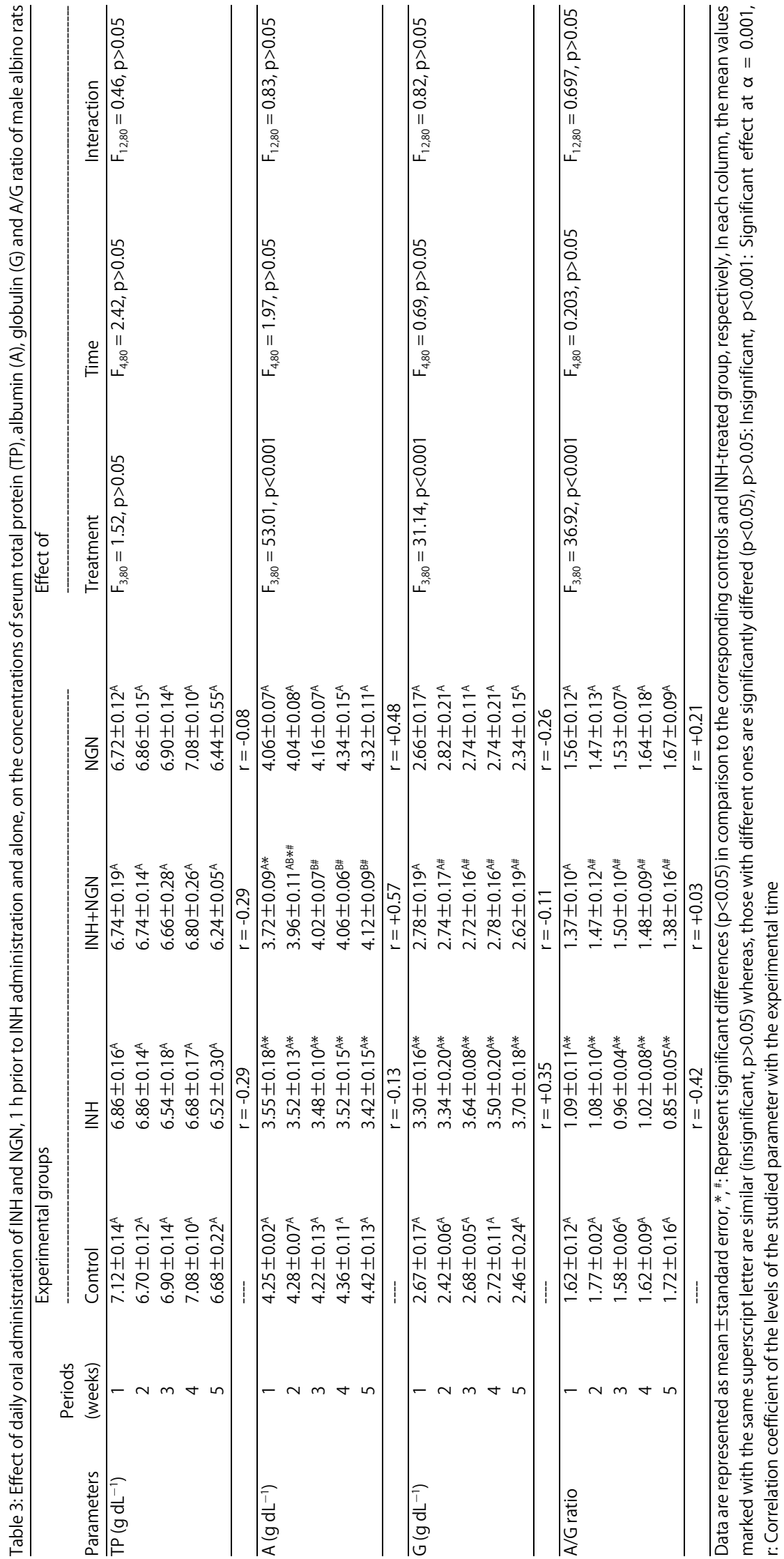




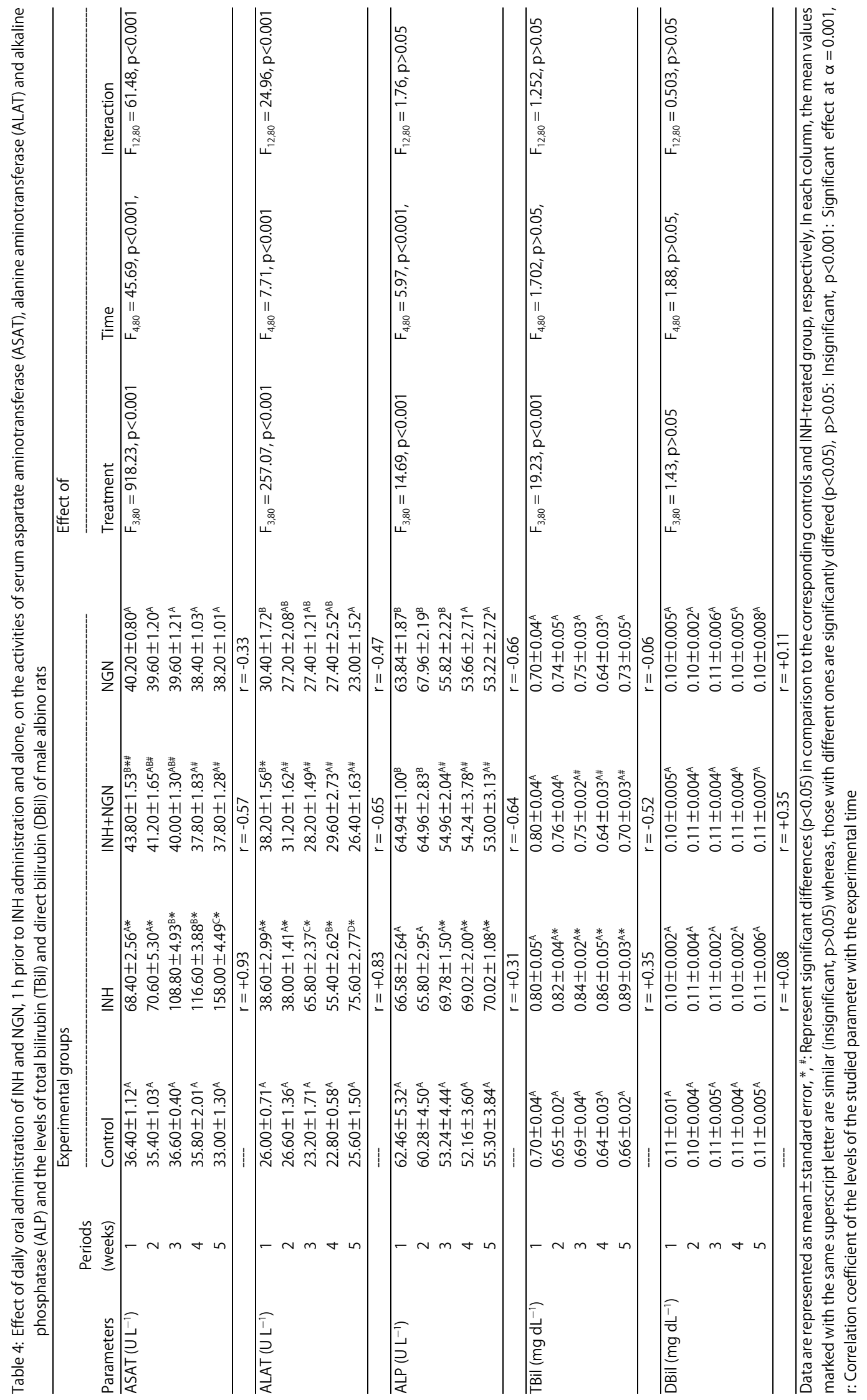




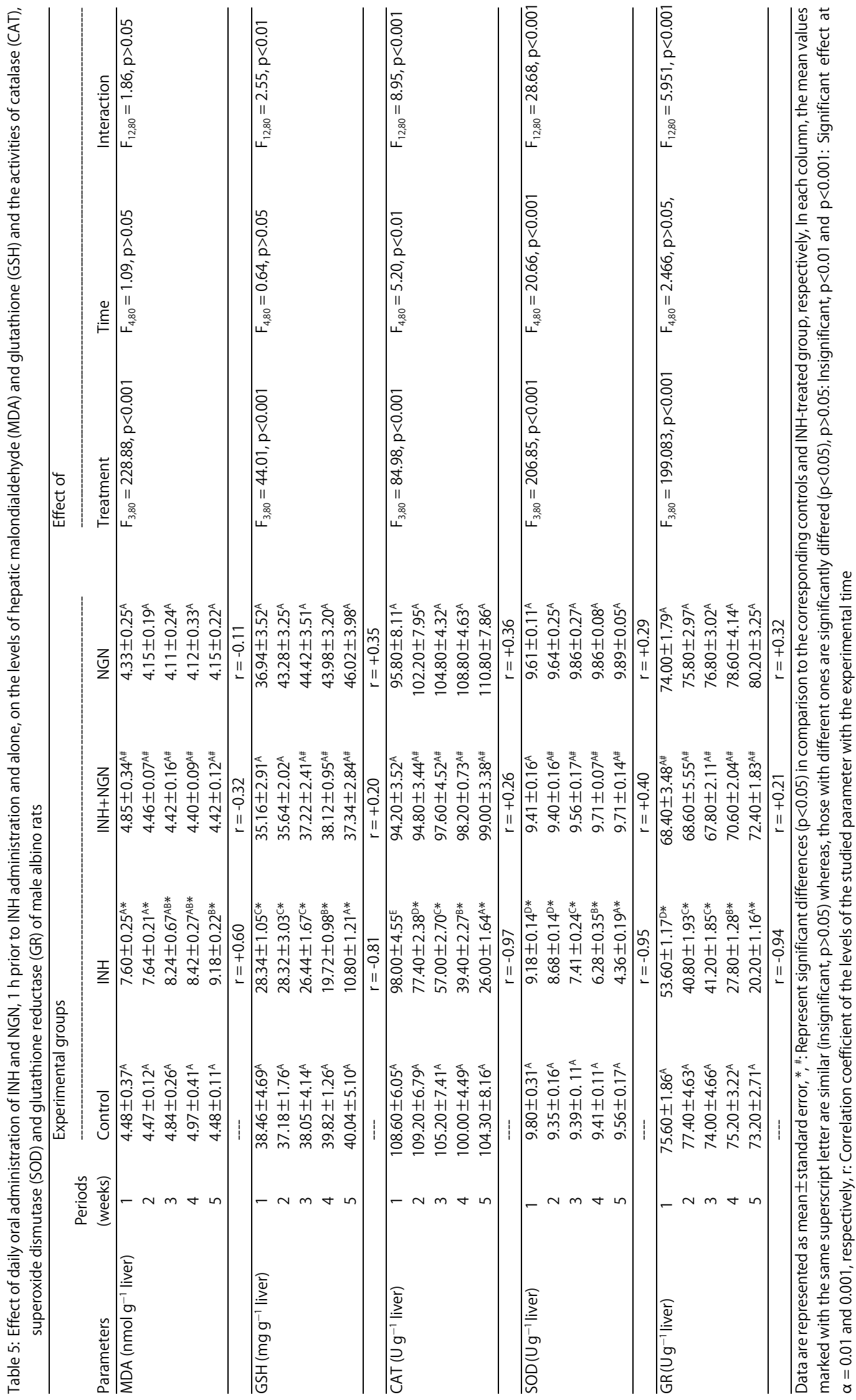


activities of CAT, SOD and GR were significantly influenced by the type of treatment. However, the experimental periods had significant effects only on the activities of CAT and SOD. In the liver of rats administered INH alone, there was a meaningful elevation in the levels of MDA accompanied by a marked reduction in the GSH content, SOD and GR activities, at all the experimental intervals, as compared to those of controls. The activity of CAT of the INH-treated group was significantly lower than that of the controls at all the experimental periods except for insignificant reduction at the 1st week. The experimental time showed a positive correlation with the levels of MDA whereas strong negative correlations were observed with the levels of GSH and the activities of CAT, SOD and $G R$, in the INH-administered group. In the rats of(NGN+INH)-treated group, the mean values of hepatic MDA concentration were significantly lower than those of $\mathrm{INH}$-treated rats and were not significantly different $(\mathrm{p}>0.05)$ from those of the controls at all the experimental periods. On the other hand, the mean values of hepatic GSH content of $(\mathrm{NGN}+\mathrm{INH})$-treated rats were significantly higher than those of INH-treated group starting from the 3rd week till the end of experiment, but they were not significantly different $(p>0.05)$ from those of controls. As compared to the INH-treated group, the rats administered NGN before INH showed a marked elevation in the activity of $G R$, from the 1st week whereas, the marked increase in the activities of CAT and SOD began form the 2 nd week till the end of the experiment. All the activities of the studied enzymatic antioxidants of (NGN+INH)-treated group did not significantly $(p>0.05)$ differ from those of the controls. The rats administered NGN alone did not exhibit any marked changes in the levels of MDA and GSH or in the activities of SOD, CAT and GR as compared with those of the corresponding controls. After administration of NGN prior to $\mathrm{INH}$ or alone, the experimental time exhibited weak negative correlations with the levels of MDA and weak positive correlations with the levels of GSH as well as the activities of enzymatic antioxidants.

\section{DISCUSSION}

The present data revealed a marked decline in the RBC and WBC counts, $\mathrm{Hb}$ content and PCV in the INH-treated rats, as compared with those of the controls. In the same line, Piso et al..$^{27}$ reported a significant depletion in the $\mathrm{Hb}$ levels and RBC count in TB patient after 4 months of treatment with INH. Similarly, marked reductions were reported in all the studied hematological parameters of male Wister rats after administration of INH in combination with RMP daily for
21 days $^{28}$. The INH-induced anemia has been categorized as pure red cell aplasia, PRCA, due to autoimmune response to nucleated $\mathrm{RBC}^{29}$ or sideroblastic anemia due to depletion of heme synthesis ${ }^{27}$. The latter authors indicated that INH-induced depletion of heme synthesis is due to inhibition of $\delta$-aminolevulinate synthase and that pyridoxine, which is a co-factor in synthesis of $\delta$-aminolevulinate, is inhibited by $\mathrm{INH}$. However, INH can cause an overproduction of free radicals leading LPO of plasma membranes and consequent rupturing of the erythrocyte membranes ${ }^{30}$. Moreover, Metushi et al. ${ }^{31}$ recorded the presence of anti-INH in the serum of INH-administered patients. These antibodies can destroy RBCs leading to a hemolytic anemia ${ }^{32}$. Regarding INH-induced leukopenia, it has been attributed mainly to the infiltration of leukocytes to the inflamed tissues $^{33}$.

On the other hand, the treatment of NGN $1 \mathrm{~h}$ prior to the administration of INH significantly ameliorated the adverse effects of INH on the studied hematological parameters. This may be explained through different mechanisms. Firstly, NGN may has induced the expression of heme oxygenase that acts as an antioxidant in the vascular cells for protection against INH-induced oxidative damage ${ }^{34}$. Secondly, NGN possibly has increased the membrane fluidity of $\mathrm{RBCs}^{35}$, preventing them from being ruptured. Thirdly, NGN could has diffused though the erythrocyte membranes and bind to the $\mathrm{Hb}$ molecules leading to their stabilization without any interference with its oxygen content ${ }^{36}$.

In the current study, the substantial elevation in the levels of TL, TC, TG and LDL-C in serum of INH-administered rats reflected the ability of $\mathrm{INH}$ to boost the lipid biosynthesis in the liver. Likewise, Pal et al. ${ }^{37}$ reported substantial elevations in the levels of TC and TL in the serum and hepatic tissue of rats following the treatment with anti-TB drugs. The enhanced lipid production may be due to the ability of INH to alter the gene expression of some enzymes that were involved in the lipid synthesis ${ }^{28}$. Moreover, INH can block the biosynthesis of $\mathrm{HDL}-\mathrm{C}$ that is responsible for the reverse transport of cholesterol from the blood back to the liver $^{38}$. This was demonstrated in the present study by the marked reduction of the HDL-C levels accompanied by a remarkable increase in the levels of TC in the INH-treated rats. On the other hand, the notable increase in the levels of the LDL-C may has increased the levels of TC in the blood through transporting more cholesterol from the liver to the blood ${ }^{39}$. The increased levels of TG that has important role in the transport and excretion of bile acids from the body ${ }^{40}$, can be translated as a mechanism to overcome the increased levels of TBil. 
The present data showed a marked alleviation in all the studied lipid profile parameters in the serum of rats administered NGN before the treatment with INH. However, the administration of NGN alone caused a momentous reduction in the levels of TL, TC and TG, as compared to the controls. Similarly, in a previous study by Alam et al. ${ }^{14}$, NGN administration caused a remarkable decline in the lipid content of male Long-Evans hooded rats. This reflects the hypolipidemic properties of NGN that successfully contracted the hyperlipidemic effect of INH. This could be due to the ability of NGN to upregulate the mRNA of many of the enzymes that are involved in $\beta$-oxidation of fats such as fatty acid synthase and acyl-CoA in the liver ${ }^{41}$. These findings confirmed the protective role of NGN against atherogenesis and heart diseases ${ }^{42}$.

In the present study, the administration of INH alone caused significant reduction in the levels of albumin concurrent with a marked elevation in the levels of globulin. Thus, the ratio of $A / G$ ratio was reduced whereas no noticeable changes in the levels of TP were recorded in the $\mathrm{INH}$-treated rats. Similar results were obtained in rats after treatment with $\mathrm{INH}$ alone ${ }^{20}$ or in combination with $\mathrm{RMP}^{43}$. The reported hypoalbuminemia could be attributed to the $\mathrm{INH}$-induced damage of hepatocyte that hinder the process of albumin synthesis in the liver ${ }^{10}$. However, the increased level of globulin reflects that the $\mathrm{INH}$-induced hepatotoxicity was immune-mediated ${ }^{44}$. Given that the INH acts as a hapten that binds to the proteins to form antigens that induce the immune system to produce antibodies against the $\mathrm{INH}^{20}$. Metushi et al. ${ }^{11}$ recorded that antibodies against the INH were detected in patients were suffering from $\mathbf{I N H}$-induced hepatotoxicity.

On contrast, the treatment with NGN, $1 \mathrm{~h}$ prior to the administration of $\mathrm{INH}$, caused a noteworthy alleviation of the protein profile parameter as shown in the present results. This was consistent with findings of Alam et al: ${ }^{14}$, who reported that NGN restored the normal levels of albumin and TP in the rats intoxicated with dimethyl nitrosamine. This reflected the role of NGN in the maintenance of normal protein synthesis. This can be due to the ability of NGN to enhance the mitochondrial enzymes in the liver cells including those of tricarboxylic acid and respiratory chain ${ }^{45}$ and consequently provided the hepatocytes with great supply of ATP required for the protein biosynthesis ${ }^{46}$.

In the present study, meaningful elevations in the serum activities of ASAT, ALAT and ALP were observed in the INH-administered rats, as compared to the controls. These findings reflect that the hepatic tissue of $\mathrm{INH}$-treated group was heavily affected. In the same direction, recent studies reported marked increases in the activities of enzymatic biomarkers of the liver functions of rats after the administration of INH alone ${ }^{20}$ or in association with RMP ${ }^{11}$. The elevated activities of ASAT and ALAT can be due to the impaired permeability of hepatocyte membranes under the action of INH and its metabolites ${ }^{21,47}$. In addition, the increased ALP activity may has increased the production of adenosine that acts as anti-inflammatory mediator for further protection of cells against the INH-induced injury ${ }^{43}$.

When the administration of INH was preceded by the treatment of NGN, the ASAT, ALAT and ALP activities remained approximately within the normal range. This indicated that the NGN has protected the hepatic tissue against INH-induced damage. Similarly, Esmaeili and Alilou ${ }^{48}$ reported a noticeable attenuation in the serum activities of ASAT and ALAT in the rats administered with NGN prior to the administration of the carbon tetrachloride. They deduced that the NGN protected the liver against the carbon tetrachloride-induced hepatotoxicity.

The levels of TBil of rats administered with INH alone exhibited a remarkable increase in their mean values while the levels of DBil were insignificantly differed $(p>0.05)$ from the controls. The results of this study were similar to those of Santhosh et $a /{ }^{43}$, who reported significant increases in the levels of serum bilirubin concurrent with marked decreases in the levels of albumin. These findings indicated that the INH affected only the unconjugated bilirubin. This can be linked to the above mentioned INH-induced hemolysis of erythrocytes and consequent breakdown of $\mathrm{Hb}$ into heme which was converted at the end into unconjugated bilirubin ${ }^{49}$. In addition, the increased levels of unconjugated bilirubin in serum may be attributed to the reduced levels of albumin required for the transport of bilirubin to the hepatocytes ${ }^{49}$.

On contrary, the levels of TBil of rats administered INH preceded by the NGN were approaching the normal levels of the controls. As previously discussed, this can be due to the ability of NGN to protect RBCs and $\mathrm{Hb}$ from the damaging effect of $\mathrm{INH}^{36}$.

In the current results, the liver of INH-treated rats showed a marked elevation in the levels of MDA accompanied by a significant depletion in the activities of enzymatic (SOD, CAT, GR) and levels of non-enzymatic (GSH) antioxidants. The present data were in the same line with many previous studies on INH and its metabolites. Abdel-Ghaffar et al.20 reported a marked increase in the MDA levels concurrent with a noticeable decrease in the SOD activity and GSH content in the liver of rats administered INH alone. Basheer et al."1 recorded significant reductions in the levels of total antioxidant capacity (TAC) and GSH but a marked elevation in 
the levels of LPO in the liver of rats after 14 days following intraperitoneal injection with $50 \mathrm{mg} \mathrm{kg}^{-1}$ b.wt., of INH and $100 \mathrm{mg} \mathrm{kg}^{-1}$ b.wt., of RMP. Santhosh et al. ${ }^{43}$ reported a significant reduction in the levels of GSH accompanied by marked elevations in the levels of MDA in liver of rats after oral administration of INH and RMP (200 $\mathrm{mg} \mathrm{kg}^{-1}$ b.wt., for each), for 1 month. Thus, oxidative stress can be considered one of the acceptable mechanisms by which INH induced hepatotoxicity. It was noteworthy that the INH can be directly hydrolyzed in the liver via amidase into hydrazine $(\mathrm{HZ})$ or subjected firstly to acetylation via $\mathrm{N}$-acetyltransferase to form acetyl-INH followed by the hydrolysis into acetyl-HZ via acyl amidase $^{50}$. The microsomal cytochrome P450 system can oxidized the $\mathrm{HZ}$ and acetyl-HZ resulting in excessive production of free radicals that induce LPO in the cellular membranes ${ }^{30}$.

On the other hand, the administration of NGN prior to the treatment with $\mathrm{INH}$ remarkably ameliorated the INH-induced variations in hepatic MDA and GSH levels and SOD, CAT and GR activities. The present results are in the same line with Renugadevi and Prabu ${ }^{21}$, Pari and Amudha ${ }^{51}$, who reported that NGN significantly reduced MDA and restored the hepatic activities of SOD, CAT, glutathione-S-transferase (GST) and glutathione peroxidase (GPX). This can be due to that the NGN possess both antioxidant and anti-inflammatory properties ${ }^{52}$. Under stress conditions, NGN directly activates the AMP kinase $^{53}$, that consequently induces the upregulation of activating transcription factor 3 resulting in attenuating the expression of several cytokines including tumor necrotic factor, TNF- $\alpha^{54}$. Thus, NGN can reduce the TNF- $\alpha$ induced generation of free radicals ${ }^{55}$. In addition, NGN has strong scavenging power of reactive oxygen species, $\mathrm{ROS}^{56}$. This can be due to the presence of polyphenolic ring in the structure of NGN that acts as an electron trap to scavenge free radicals ${ }^{57}$. In vitro studies showed that NGN markedly attenuated the activities of xanthine oxidase which acts as a physiological source of ROS ${ }^{14}$. Furthermore, the NGN has inhibitory effects on the cytochrome P450 enzymes ${ }^{14}$, that are responsible for the metabolism and bioactivation of INH. NGN may has upregulated the nuclear factor erythroid related factor 2 (Nrf2) which is involved in the transcription of many endogenous antioxidant genes including CAT, SOD, GST, GPx and heme oxygenase, $\mathrm{HO}^{-1}{ }^{52}$. The $\mathrm{NGN}$ may has also improved the expression of glutamate cysteine ligase required for the synthesis of GSH ${ }^{52}$. Moreover, inhibition of ROS toxicity by NGN has shown to be effective in preventing caspase activation in $\mathrm{CCl}_{4}$-induced hepatotoxicity ${ }^{57,58}$.

\section{CONCLUSION}

The present results showed that the administration of $\mathrm{INH}$ alone can induce hepatotoxicity mediated by the oxidative damage as manifested by the increased level of hepatic MDA that was accompanied by significant depletion in the hepatic content of endogenous antioxidants in a time-dependent manner. However, the administration of NGN prior to the treatment with $\mathrm{INH}$ has protected the liver and effectively barred the adverse actions of the INH on all the studied hematological and biochemical parameters. Moreover, the treatment with NGN alone did not induce any significant changes in all the studied parameters, as compared to the controls except for a marked reduction in the levels of $T L, T C$ and LDL-C that highlights its hypolipidemic action.

\section{SIGNIFICANCE STATEMENT}

The current study throws light on the mechanism by which NGN can reverse the INH-linked toxicity. This will pave the way for incorporating the NGN with the anti-TB drugs during the treatment regimens, in the future.

\section{REFERENCES}

1. Bregani, E.R., C. Valcarenghi, T.van Tien and V. Monzani, 2013. Suggestive criteria for pulmonary tuberculosis in developing countries. Int. J. Mycobacteriol., 2: 211-213.

2. Dalvin, L.A. and W.M. Smith, 2017. Intraocular manifestations of mycobacterium tuberculosis: A review of the literature. J. Clin. Tuberc. Other Mycobact. Dis., 7: 13-21.

3. Mitchison, D. and G. Davies, 2013. The chemotherapy of tuberculosis: past, present and future [State of the art]. Int. J. Tuberc. Lung Dis., 16: 724-732.

4. Sia, I.G. and M.L. Wieland, 2011. Current concepts in the management of tuberculosis. Mayo Clin. Proc., 86: 348-361.

5. Metushi, I.G., P. Cai, X. Zhu, T. Nakagawa and J.P. Uetrecht, 2011. A fresh look at the mechanism of isoniazid-induced hepatotoxicity. Clin. Pharmacol. Ther., 89: 911-914.

6. Souayed, N., M. Chennoufi, N.B. Frej, A. Chaabane and M. Ben-Attia et al., 2016. Circadian variation of isoniazid pharmacokinetics in mice. Biomed. Pharmacother., 84: 1150-1155.

7. Tafazoli, S., M. Mashregi and P.J. O'Brien, 2008. Role of hydrazine in isoniazid-induced hepatotoxicity in a hepatocyte inflammation model. Toxicol. Applied Pharmacol., 229: 94-101. 
8. Quinlan, C.L., A.L. Orr, I.V. Perevoshchikova, J.R. Treberg and B.A. Ackrell and M.D. Brand, 2012. Mitochondrial complex II can generate reactive oxygen species at high rates in both the forward and reverse reactions. J. Biol. Chem., 287: 27255-27264.

9. Rutter, J., D.R. Winge and J.D. Schiffman, 2010. Succinate dehydrogenase-assembly, regulation and role in human disease. Mitochondrion, 10: 393-401.

10. Eminzade, S., F. Uras and F.V. Izzettin, 2008. Silymarin protects liver against toxic effects of anti-tuberculosis drugs in experimental animals. Nutr. Metab., Vol. 5. 10.1186/17437075-5-18.

11. Basheer, A.S., A. Siddiqui, Y.N. Paudel, M.Q. Hassan, M. Imran, A.K. Najmi and M. Akhtar, 2017. Hepatoprotective and antioxidant effects of fish oil on isoniazid-rifampin induced hepatotoxicity in rats. Pharma Nutr., 5: 29-33.

12. Meydani, M. and S.T. Hasan, 2010. Dietary polyphenols and obesity. Nutrients, 2: 737-751.

13. Ke, J., K.L. Kliewer, E.M. Hamad, R.M. Cole and K.A. Powell et al., 2015. The flavonoid, naringenin, decreases adipose tissue mass and attenuates ovariectomy-associated metabolic disturbances in mice. Nutr. Metab., Vol. 12. 10.1186/1743-7075-12.

14. Alam, M.A., N. Subhan, M.M. Rahman, S.J. Uddin, H.M. Reza and S.D. Sarker, 2014. Effect of citrus flavonoids, naringin and naringenin, on metabolic syndrome and their mechanisms of action. Adv. Nutr. Int. Rev. J., 5: 404-417.

15. Qin, W., B. Ren, S. Wang, S. Liang and B. He et al., 2016. Apigenin and naringenin ameliorate PKC $\beta$ Il-associated endothelial dysfunction via regulating ROS/caspase-3 and NO pathway in endothelial cells exposed to high glucose. Vasc. Pharmacol., 85: 39-49.

16. Bansal, P., P. Paul, J. Mudgal, P.G. Nayak, S.T. Pannakal, K.I. Priyadarsini and M.K. Unnikrishnan, 2012. Antidiabetic, antihyperlipidemic and antioxidant effects of the flavonoid rich fraction of Pilea microphylla (L.) in high fat diet/streptozotocin-induced diabetes in mice. Exp. Toxicol. Pathol., 64: 651-658.

17. Hasanein, P. and F. Fazeli, 2014. Role of naringenin in protection against diabetic hyperalgesia and tactile allodynia in male Wistar rats. J. Physiol. Biochem., 70: 997-1006.

18. Anca, H., A. Aurel, S. Miruna, H. Nicoleta, M. Ciprian-Valentin, C. Marieta and D. Anca, 2014. Antioxidant and hepatoprotective effects of naringenin and its $\beta$-Cyclodextrin formulation in mice intoxicated with carbon tetrachloride: $A$ comparative study. J. Med. Food, 17: 670-677.

19. Mershiba, S.D., M.V. Dassprakash and S.D. Saraswathy, 2013. Protective effect of naringenin on hepatic and renal dysfunction and oxidative stress in arsenic intoxicated rats. Mol. Biol. Rep., 40: 3681-3691.

20. Abdel-Ghaffar, O., S.T. Mahmoud, A.A. Said and F.A.Y. Sanad, 2017. Hepatoprotective effect of rutin against oxidative stress of Isoniazid in albino rats. Int. J. Pharmacol., 13: 516-528.
21. Renugadevi, J. and S.M. Prabu, 2010. Cadmium-induced hepatotoxicity in rats and the protective effect of naringenin. Exp. Toxicol. Pathol., 62: 171-181.

22. Ohkawa, H., N. Ohishi and K. Yagi, 1979. Assay for lipid peroxides in animal tissues by thiobarbituric acid reaction. Anal. Biochem., 95: 351-358.

23. Beutler, E., O. Duron and B.M. Kelly, 1963. Improved method for the determination of blood glutathione. J. Lab. Clin. Med., 61: 882-888.

24. Aebi, H., 1984. Catalase in vitro. Methods Enzymol., 105: 121-126.

25. Nishikimi, M., N.A. Roa and K. Yogi, 1972. The occurrence of superoxide anion in the reaction of reduced phenazine methosulfate and molecular oxygen. Biochem. Biophys. Res. Commun., 46: 849-854.

26. Goldberg, D.M. and R.J. Spooner, 1983. Method for the determination of glutathione reductase. Methods Enzymatic Anal., 3: 258-265.

27. Piso, R.J., K. Kriz and M.C. Desax, 2011. Severe isoniazid related sideroblastic anemia. Hematol. Rep., Vol. 3. 10.4081/hr.2011.e2.

28. Arundhathi, S., A.A. Kumar, Y.R. Kumar and B.A. Kumar, 2015. Haematological and histopathological alterations due to combined toxicity of isoniazid and rifampicin; amelioration with Withania somnifera and vitamin-E in Wistar rats. Int. J. Pharma Bio. Sci., 6: 222-229.

29. Loulergue, P., O. Mir and R. Dhote, 2007. Pure red blood cell aplasia and isoniazid use. Emerg. Infect. Dis., 13: 1427-1428.

30. Ramappa, V. and G.P. Aithal, 2013. Hepatotoxicity related to anti-tuberculosis drugs: Mechanisms and management. J. Clin. Exp. Hepatol., 3: 37-49.

31. Metushi, I.G., C. Sanders, W.M. Lee and J. Uetrecht, 2014. Detection of anti-isoniazid and anti-cytochrome P450 antibodies in patients with isoniazid-induced liver failure. Hepatology, 59: 1084-1093.

32. Mintzer, D.M., S.N. Billet and L. Chmielewski, 2009. Drug induced hematologic syndromes. Adv. Hematol., 10.1155/2009/495863.

33. Abdel-Ghaffar, O., S.T. Mahmoud, A.A. Said and F.A.A Youssef Sanad, 2017. Ameliorative effect of rutin against isoniazid-induced alterations in certain hematological and biochemical parameters of albino rats. Int. J. Pharmacol., (In Press).

34. Nyane, N.A., T.B. Tlaila, T.G. Malefane, D.E. Ndwandwea and P.M.O. Owira, 2017. Metformin-like antidiabetic, cardio-protective and non-glycemic effects of naringenin: Molecular and pharmacological insights. Eur. J. Pharmacol., 803: 103-111.

35. Ajdzanovic, A., V. Jakovljevic, D. Milenkovic, A. Konic-Ristic, J. Zivanovic, I. Jaric and V. Milosevic, 2015. Positive effects of naringenin on near-surface membrane fluidity in human erythrocytes. Acta Physiol. Hung., 102: 131-136. 
36. Maity, S., S. Chakraborty and A.S. Chakraborti, 2017. Critical insight into the interaction of naringenin with human haemoglobin: A combined spectroscopic and computational modeling approaches. J. Mol. Struct., 1129: 256-262.

37. Pal, R., S.V. Rana, K. Vaiphei and K. Singh, 2008. Isoniazid-rifampicin induced lipid changes in rats. Clin. Chim. Acta, 389: 55-60.

38. Annema, W. and U.J.F. Tietge, 2012. Regulation of reverse cholesterol transport-a comprehensive appraisal of available animal studies. Nutr. Metabol., Vol. 9. 10.1186/1743-7075-9-25.

39. Harisa, G.I. and F.K. Alanazi, 2014. Low density lipoprotein bionanoparticles: From cholesterol transport to delivery of anti-cancer drugs. Saudi Pharm. J., 22: 504-515.

40. Cheng, J., K.W. Krausz, F. Li, X. Ma and F.J. Gonzalez, 2013. CYP2E1-dependent elevation of serum cholesterol, triglycerides and hepatic bile acids by isoniazid. Toxicol. Applied Pharmacol., 266: 245-253.

41. Fukuchi, Y., M. Hiramitsu, M. Okada, S. Hayashi, Y. Nabeno, T. Osawa and M. Naito, 2008. Lemon polyphenols suppress diet-induced obesity by up-regulation of mRNA levels of the enzymes involved in $\beta$-oxidation in mouse white adipose tissue. J. Clin. Biochem. Nutr., 43: 201-209.

42. Chtourou, Y., A.B. Slima, M. Makni, R. Gdoura and H. Fetoui, 2015. Naringenin protects cardiac hypercholesterolemia-induced oxidative stress and subsequent necroptosis in rats. Pharmacol. Rep., 67: 1090-1097.

43. Santhosh, S., T.K. Sini, R. Anandan and P.T. Mathew, 2007. Hepatoprotective activity of chitosan against isoniazid and rifampicin-induced toxicity in experimental rats. Eur. J. Pharmacol., 572: 69-73.

44. Metushi, I.G. and J. Uetrecht, 2014. Isoniazid-induced liver injury and immune response in mice. J. Immunotoxicol., 11: 383-392.

45. Rajadurai, M. and P.S.M. Prince, 2007. Preventive effect of naringin on cardiac mitochondrial enzymes during isoproterenol-induced myocardial infarction in rats: A transmission electron microscopic study. J. Biochem. Mol. Toxicol., 21: 354-361.

46. Jewett, M.C., M.L. Miller, Y. Chen and J.R. Swartz, 2009. Continued protein synthesis at low [ATP] and [GTP] enables cell adaptation during energy limitation. J. Bacteriol., 191: 1083-1091.

47. Ahmad, F. and N. Tabassum, 2012. Experimental models used for the study of antihepatotoxic agents. J. Acute Dis., 1:85-89.
48. Esmaeili, M.A. and M. Alilou, 2014. Naringenin attenuates $\mathrm{CCl}_{4}$-induced hepatic inflammation by the activation of an Nrf2-mediated pathway in rats. Clin. Exp. Pharmacol. Physiol., 41: 416-422.

49. Jayachandra, K. and V.D. Devi, 2012. Development of herbal drugs in the treatment of Jaundice-An overview. Int. J. Ayurvedic Herb. Med., 2: 636-645.

50. Wang, P., K. Pradhan, X.B. Zhong and X. Ma, 2016. Isoniazid metabolism and hepatotoxicity. Acta Pharm. Sin. B, 6: 384-392.

51. Pari, L. and K. Amudha, 2011. Hepatoprotective role of naringin on nickel-induced toxicity in male Wistar rats. Eur. J. Pharmacol., 650: 364-370.

52. Ramprasath, T., M. Senthamizharasi, V. Vasudevan, S. Sasikumar, S. Yuvaraj and G.S. Selvam, 2014. Naringenin confers protection against oxidative stress through upregulation of $\mathrm{Nrf}_{2}$ target genes in cardiomyoblast cells. J. Physiol. Biochem., 70: 407-415.

53. Wiejak, J., J. Dunlop, S.P. Mackay and S.J. Yarwood, 2013. Flavanoids induce expression of the suppressor of cytokine signalling 3 (SOCS3) gene and suppress IL-6-activated signal transducer and activator of transcription 3 (STAT3) activation in vascular endothelial cells. Biochem. J., 454: 283-293.

54. Liu, X., N. Wang, S. Fan, X. Zheng and Y. Yang et al., 2016. The citrus flavonoid naringenin confers protection in a murine endotoxaemia model through AMPK-ATF3-dependent negative regulation of the TLR4 signalling pathway. Scient. Rep., Vol. 6. 10.1038/srep39735

55. Chen, S., Y. Ding, W. Tao, W. Zhang, T. Liang and C. Liu, 2012. Naringenin inhibits TNF- $\alpha$ induced VSMC proliferation and migration via induction of HO-1. Food Chem. Toxicol., 50: 3025-3031.

56. Cavia-Saiz, M., M.D. Busto, M.C. Pilar-Izquierdo, N. Ortega, M. Perez-Mateos and P. Muniz, 2010. Antioxidant properties, radical scavenging activity and biomolecule protection capacity of flavonoid naringenin and its glycoside naringin: $A$ comparative study. J. Sci. Food Agric., 90: 1238-1244.

57. Ahmed, L.A., A.A. Obaid, H.F. Zaki and A.M. Agha, 2014. Naringenin adds to the protective effect of I-arginine in monocrotaline-induced pulmonary hypertension in rats: Favorable modulation of oxidative stress, inflammation and nitric oxide. Eur. J. Pharm. Sci., 62: 161-170.

58. Yen, F.L., T.H. Wu, L.T. Lin, T.M. Cham and C.C. Lin, 2009. Naringenin-loaded nanoparticles improve the physicochemical properties and the hepatoprotective effects of naringenin in orally-administered rats with $\mathrm{CCl}_{4}$-induced acute liver failure. Pharm. Res., 26: 893-902. 